\title{
Presencia suburbana de Puma concolor (Carnivora: Felidae) en el cantón de San Ramón, Alajuela, Costa Rica
}

\section{Brayan Heiner Morera-Chacón Yaina Ruiz-Chaves}

Gestión de los Recursos Naturales. Universidad de Costa Rica, Sede de Occidente. morera.b91@ gmail.com

El Puma (Puma concolor) es una especie que se encuentra distribuida desde Canadá hasta el Sur de Argentina y Chile, siendo común en casi todas las áreas protegidas de Costa Rica con más de 10.000 ha de extensión (Gutiérrez et al. 2015). Sin embargo, los avistamientos de Puma han sido poco documentados en el cantón de San Ramón, ubicado en la región occidental de Costa Rica. Estos reportes han sido en su mayoría al centro y norte del cantón, y usualmente dentro de grandes zonas boscosas (Cartín 2011). En este trabajo reportamos la presencia de Puma en cercanías de la ciudad de San Ramón en Costa Rica.

Algunas especies de carnívoros de tamaño pequeño y mediano se han adaptado a sitios perturbados (Crooks 2002); sin embargo, a pesar de que el Puma es una especie de gran tamaño, es cada vez más común observarlo en zonas alteradas y paisajes agrícolas cerca de las ciudades (e.g. Arias-Alzate et al. 2015, Mazzolli 2012, Pardo et al. 2017). Las variables que más influyen en la distribución de la especie son: las actividades humanas, la cobertura vegetal y la presencia de cuerpos de agua (Minjarez 2013, Gutiérrez et al. 2015); estos factores pueden cambiar a través del tiempo y podrían determinar la presencia, ausencia o recolonización del Puma en un sitio. En sitios donde el Puma es simpátrico con el Jaguar (Panthera onca), se han observado diferencias en la selección de presas, siendo el Puma la especie que en promedio consume presas más pequeñas (Estrada 2008, Hernández 2014). En Costa Rica se ha registrado la presencia de presas pequeñas en la dieta del Puma tales como el Zorro Pelón (Didelphis marsupialis), la Guatusa (Dasyprocta punctata), y el Pizote (Nasua narica; Chinchilla 1997, Bustamante et al. 2009), especies comunes en algunas zonas suburbanas (cercanas a ciudades).

El 19 de marzo de 2016 a las 14h50 observamos un Puma (Figura 1) en un parche de bosque pequeño (5,2 ha) en el distrito de Santiago, San Ramón, Provincia de Alajuela, Costa Rica (10,057109 N, -84,492416 W; Figura 2). Según el sistema de clasificación de zonas de vida de Holdridge (Holdridge 1987) el sitio de observación pertenece a un bosque muy húmedo premontano, y está ubicado a una altitud de $1160 \mathrm{msnm}$. Este parche de bosque se encuentra cercano a plantaciones de café y caseríos, donde se pueden observar frecuentemente algunas presas potenciales como el Zorro Pelón y la Guatusa. La distancia lineal desde el sitio de la observación a la ciudad de San Ramón (la más cercana) es de 4,13 km y de 2,32 km hasta la autopista, por lo que consideramos que el registro proviene de una zona suburbana.

La urbanización y deforestación de hábitats naturales, así como las actividades asociadas al transporte motorizado, representan las mayores amenazas para esta especie (Arias-Alzate et al. 2015). Reportes como este demuestran que los parches de bosque pequeños cerca a zonas urbanas son importantes, ya que pueden funcionar como refugios temporales para la especie en su desplazamiento a hábitats idóneos y/o para protegerse de cazadores.

\section{Referencias}

ARIAS-ALZATE, A. et al. 2015. Presencia de Puma (Puma concolor) en un paisaje periurbano al sur del Valle de Aburrá, Antioquia, Colombia. Mammalogy Notes 2(1): 24-28. BUSTAMANTE-H O., et al. 2009. Depredación de un pizote (Nasua narica) por un puma (Puma concolor) en el sureste de la Península de Osa, Costa Rica. Acta Biol. Panamensis 1: 39-45. CARTÍN, M. 2011. Posible exclusión competitiva entre pumas y jaguares (Carnivora: Felidae) de la Reserva Biológica Alberto Manuel Brenes, San Ramón, Costa Rica. Brenesia 75-76: 115-117. CHINCHILLA, F. A. 1997. La dieta del jaguar (Panthera onca), el puma (Puma concolor) y el manigordo (Felis pardalis) (Carnivora; Felidae) en el Parque Nacional Corcovado, Costa Rica. Revista de Biología Tropical 45: 1223-1230.

CROOKS, K. R. 2002. Relative sensitivities of mammalian carnivores to habitat fragmentation. Conservation Biology 16: 488-502.

ESTRADA, C. G. 2008. Dieta, uso de hábitat y patrones de actividad del puma (Puma concolor) y el jaguar (Panthera onca) en la selva maya. Revista Mexicana de Mastozoología 12(1): 113-130. GUTIÉRREZ, A., et al. 2015. Felinos de Costa Rica: compendio de investigaciones realizadas en la UNA. Universidad Nacional. Heredia. Costa Rica 113.

HERNÁNDEZ, A. D. 2014. Ecología del jaguar (Panthera onca) y el puma (Puma concolor) en la reserva de la biósfera Sierra del Abra-Tanchipa, México. Tesis de Doctorado. Institución de Enseñanza e Investigaciones en Ciencias Agrícolas, Salinas de Hidalgo 74.

HOLDRIDGE, L. R. 1987. Ecología basada en zonas de vida. Agroamérica 83.

MAZZOLLI, M. 2012. Natural recolonization and suburban presence of pumas (Puma concolor) in Brazil. Journal of Ecology and the Natural Environment 4(14): 344-362.

MINJAREZ, A. 2013. Análisis de la distribución del puma (Puma concolor) en Sierra La Giganta, Baja California Sur. Tesis de Maestría. Centro de Investigaciones Biológicas del Noroeste, S.C. 88.

PARDO, L.E., et al. 2017. Registros de puma (Puma concolor, Linnaeus, 1771) en zona rural de San Carlos de Guaroa, Meta, Colombia. Rev. Biodiversidad Neotropical 7(1): 56-61.

\section{Mammalogy Notes | Notas Mastozoológicas \\ Sociedad Colombiana de Mastozoología \\ Vol. 4 Num. 2| 2018}


MAMMALOGY NOTES| NOTAS MASTOZOOLÓGICAS

ISSN 2382-3704

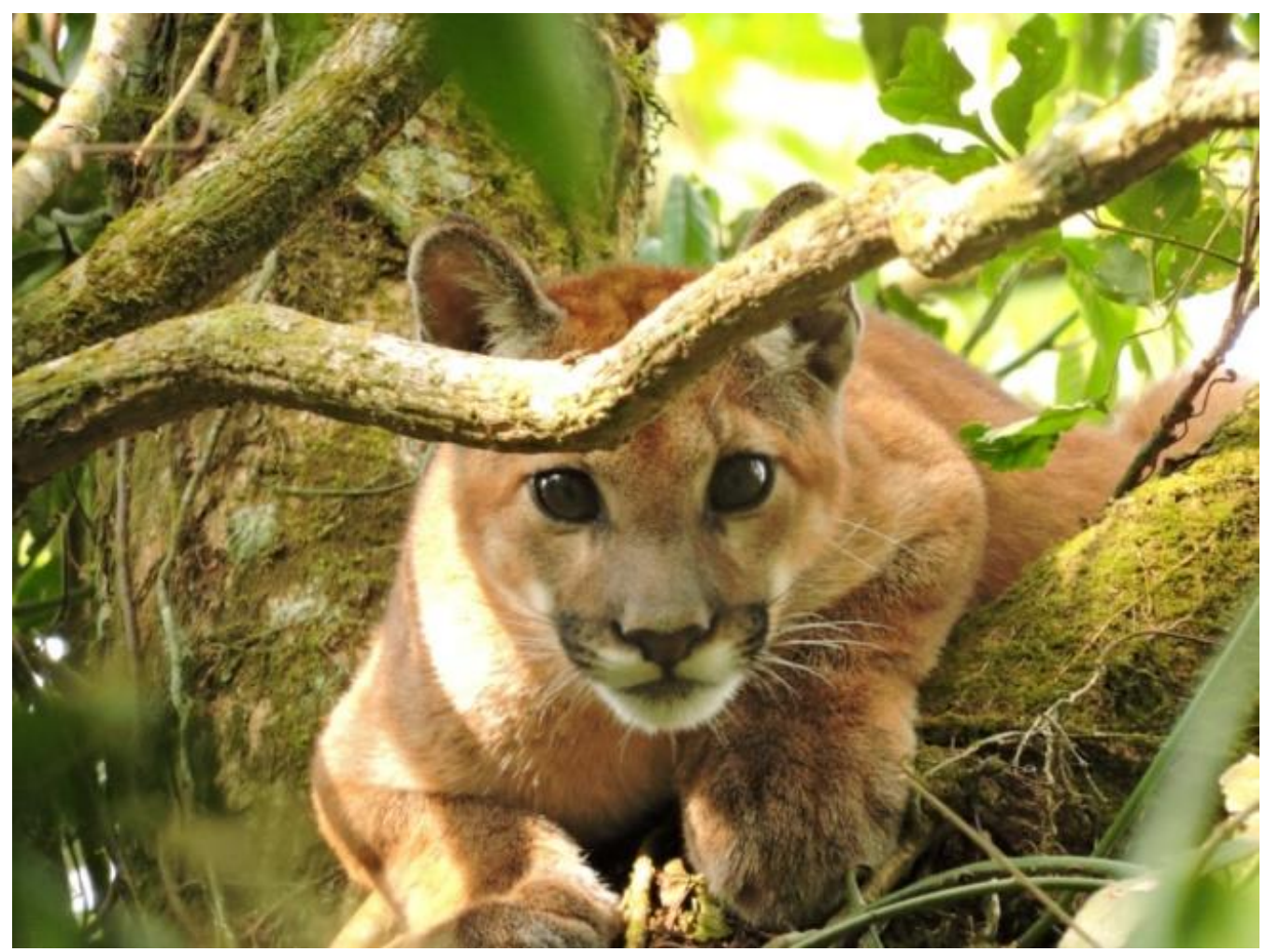

Figura 1. Fotografía de Puma (Puma concolor) en el distrito de Santiago, San Ramón, Provincia de Alajuela, Costa Rica. Foto: Yaina Ruiz.

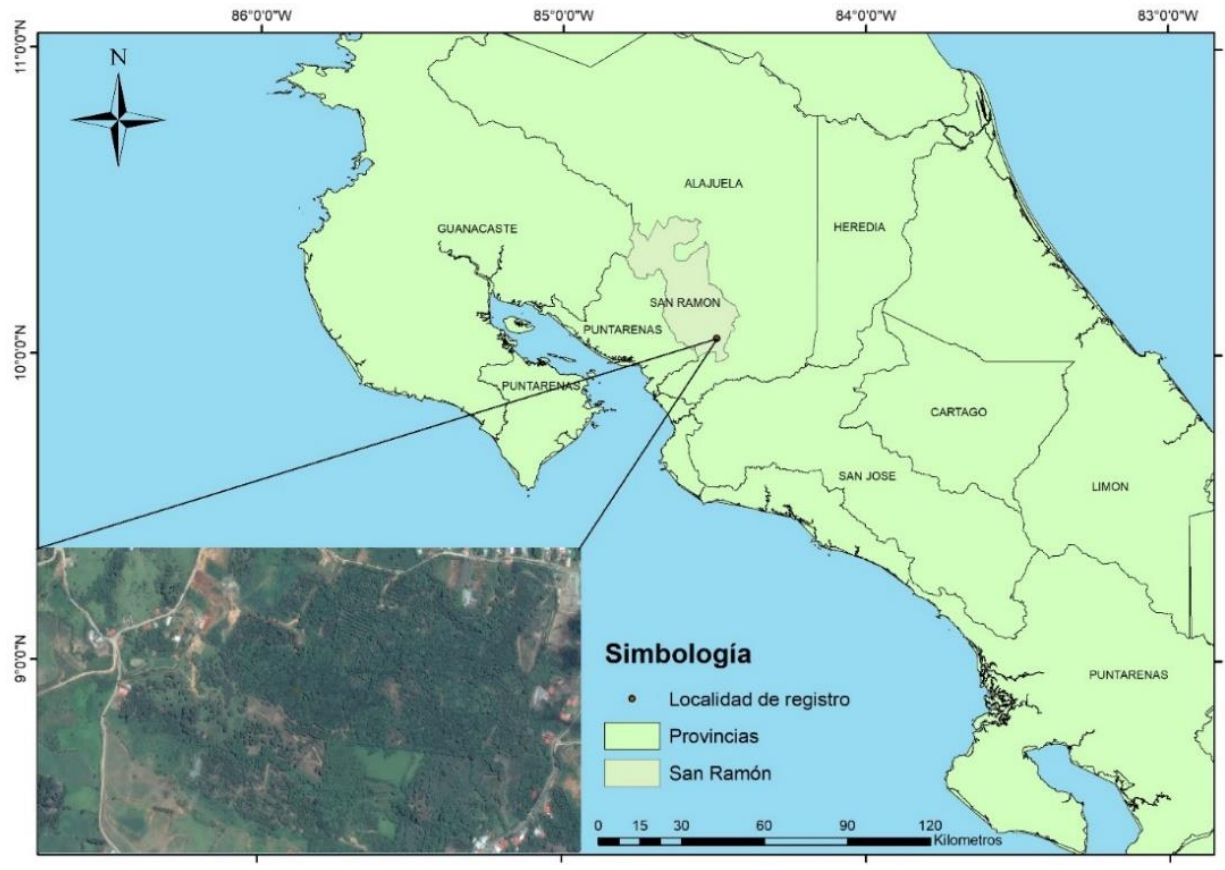

Figura 2. Localidad de registro de Puma (Puma concolor) en el Cantón de San Ramón, Provincia Alajuela, Costa Rica.

\author{
Mammalogy Notes | Notas Mastozoológicas \\ Sociedad Colombiana de Mastozoología \\ Vol. 4 Num. 2| 2018
}

